\title{
A prognostic tool to predict outcomes in children undergoing the Norwood operation
}

\author{
Punkaj Gupta, MBBS, ${ }^{\mathrm{a}}$ Avishek Chakraborty, PhD, ${ }^{\mathrm{b}}$ Jeffrey M. Gossett, MS, ${ }^{\mathrm{c}}$ and \\ Mallikarjuna Rettiganti, $\mathrm{PhD}^{\mathrm{c}}$
}

\section{ABSTRACT}

Objectives: To create and validate a prediction model to assess outcomes associated with the Norwood operation.

Methods: The public-use dataset from a multicenter, prospective, randomized single-ventricle reconstruction trial was used to create this novel prediction tool. A Bayesian lasso logistic regression model was used for variable selection. We used a hierarchical framework by representing discrete probability models with continuous latent variables that depended on the risk factors for a particular patient. Bayesian conditional probit regression and Markov chain Monte Carlo simulations were then used to estimate the effects of the predictors on the means of these latent variables to create a score function for each of the study outcomes. We also devised a method to calculate the risk of outcomes associated with the Norwood operation before the actual heart operation. The 2 study outcomes evaluated were in-hospital mortality and composite poor outcome.

Results: The training dataset used 520 patients to generate the prediction model. The model included patient demographics, baseline characteristics, cardiac diagnosis, operation details, site volume, and surgeon experience. An online calculator for the tool can be accessed at https://soipredictiontool.shinyapps.io/ NorwoodScoreApp/. Model validation was performed on 520 observations using an internal 10-fold cross-validation approach. The prediction model had an area under the curve of 0.77 for mortality and 0.72 for composite poor outcome on the validation dataset.

Conclusions: Our new prognostic tool is a promising first step in creating realtime risk stratification in children undergoing a Norwood operation; this tool will be beneficial for the purposes of benchmarking, family counseling, and research. (J Thorac Cardiovasc Surg 2017;154:2030-7)

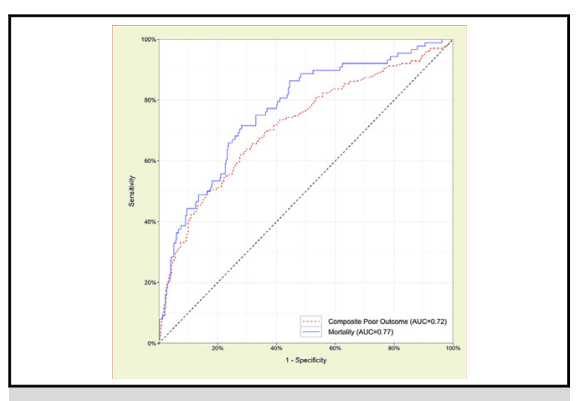

Receiver operating characteristic curves

\section{Central Message}

We report a novel prognostic tool to predict real-time outcomes among children undergoing the Norwood operation. The tool can be used for benchmarking, family counseling, and research.

\section{Perspective}

Our novel prognostic tool is a first step in creating real-time risk stratification for children undergoing a Norwood operation. This tool provides efficient risk-adjustment by condensing 10 risk factors into 1 probability before the heart operation and condensing 18 risk factors into 1 probability after the operation; this prognostic tool predicts poor outcomes associated with the Norwood operation.

See Editorial Commentary page 2038.
Patients with single-ventricle lesions and systemic outflow obstruction undergo palliation with the Norwood operation. Unfortunately, this procedure is associated

\footnotetext{
From the a Division of Pediatric Cardiology, Department of Pediatrics, University of Arkansas for Medical Sciences, Arkansas Children's Hospital, Little Rock; ${ }^{b}$ Department of Mathematical Sciences, University of Arkansas, Fayetteville; and ${ }^{c}$ Biostatistics Program, Department of Pediatrics, University of Arkansas for Medical Sciences, Arkansas Children's Hospital Research Institute, Little Rock, Ark.

Received for publication Feb 16, 2017; revisions received July 18, 2017; accepted for publication Aug 7, 2017; available ahead of print Sept 20, 2017.

Address for reprints: Punkaj Gupta, MBBS, Division of Pediatric Cardiology, Department of Pediatrics, University of Arkansas for Medical Sciences, College of Medicine, Arkansas Children's Hospital, 1 Children's Way, Slot 512-3, Little Rock, AR 72223 (E-mail: pgupta2@uams.edu).

$0022-5223 / \$ 36.00$

Copyright (c) 2017 by The American Association for Thoracic Surgery

http://dx.doi.org/10.1016/j.jtcvs.2017.08.034
}

with high morbidity and mortality. ${ }^{1-4}$ There are published data that report the risk factors associated with poor outcomes after this high-risk heart operation $^{1,5-7}$; however, there is no prognostic scoring system that can predict outcomes for an individual patient after a Norwood operation. As clinical judgment alone can be imperfect, prognostic scoring systems can be used as adjuncts to clinical decision-making in the care of individual patients. 8,9

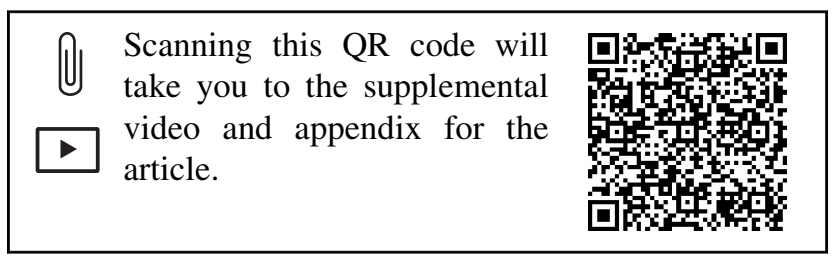




\section{Abbreviations and Acronyms \\ ECMO $=$ extracorporeal membrane oxygenation \\ MBT = modified Blalock-Taussig \\ MCMC $=$ Markov chain Monte Carlo \\ RVPA = right ventricle-pulmonary artery \\ SVR = Single-Ventricle Reconstruction}

Therefore, we sought to create a prediction tool to assess outcomes associated with the Norwood operation both before and after the operation; to do this, we used advanced statistical models, such as Bayesian conditional probit regression models and Markov chain Monte Carlo (MCMC) simulations. The 2 specific outcomes evaluated in our study were in-hospital mortality and composite poor outcome (defined as mortality or heart transplantation or need for cardiopulmonary resuscitation or use of extracorporeal membrane oxygenation [ECMO] outside the operating room or prolonged mechanical ventilation or prolonged hospital length of stay). The purpose of creating this tool was to provide families and health care providers with realistic expectations both before and after the Norwood operation; this tool will also help with benchmarking, quality improvement, cost reduction, and observational research.

\section{MATERIAL AND METHODS Data Source}

The Single Ventricle Reconstruction (SVR) Trial was a prospective study that compared outcomes between patients who underwent the Norwood operation. The subjects were randomized to a modified BlalockTaussig (MBT) shunt or a right ventricle-pulmonary artery (RVPA) shunt at the time of the Norwood operation. Details of the trial design and primary results have been reported. ${ }^{10,11}$ The trial was performed at 15 centers in the United States and Canada between 2005 and 2009, and a public-use data set from the SVR trial became available in 2013. The 2 specific outcomes evaluated in our study were in-hospital mortality and composite poor outcome. The 75th percentile of the cohort data distribution was used to establish cutoff values for defining prolonged mechanical ventilation and prolonged hospital length of stay. This definition uses the same approach as Wernovsky and colleagues. ${ }^{12}$ The University of Arkansas for Medical Sciences Institutional Review Board for the protection of human subjects reviewed the study protocol and determined that querying deidentified patient data does not fall under the jurisdiction of the Institutional Review Board oversight process.

\section{Study Population}

Patients undergoing Norwood operation for a diagnosis of hypoplastic left heart syndrome or a related single, morphologic right ventricular anomaly from the Pediatric Heart Network SVR trial public use dataset were included. ${ }^{10,11}$ No specific exclusion criteria were applied for the patients included in the SVR trial public-use dataset. The original dataset excluded patients with cardiac anatomy that would render either the MBT shunt or the RVPA shunt technically impossible; it also excluded patients with any major congenital or acquired extracardiac abnormality. Data collected included demographic information, baseline characteristics, anatomic diagnoses, interventions performed before Norwood operation, operation characteristics, and center data.

\section{Analysis and Model Creation}

The primary goal of this study was to use a model-based approach to create and validate a prediction tool for each of the 2 outcomes among patients undergoing the Norwood operation (both before and after the operation). Variables with missing data were imputed once by fully conditional specification before variable selection.

Variable selection. We used a Bayesian lasso logistic regression model to perform variable selection and regularization to create the prediction tool. Several candidate preoperative and operative predictors were used jointly to predict composite poor outcome in the variable selection model. For shrinkage, a lasso prior was used on the coefficients. The MCMC-based estimation for the Bayesian lasso logistic regression model was performed with the bayesreg package in R (R Core Team, Vienna, Austria). ${ }^{13}$ After MCMC simulations, we obtained posterior samples for each of the coefficients and constructed the middle $50 \%$ posterior credible sets. Predictors were considered to be significant only if corresponding credible sets did not include 0 . Other predictors were selected for inclusion a priori due to clinical importance or based on the literature.

Prediction model. We used Bayesian conditional probit regression and MCMC simulations from the posterior distribution of the parameters to create a prediction tool for the study outcomes. To eliminate the effect of the scale of the continuous variables on the score, we standardized each continuous variable to a $[0,1]$ range by subtracting the minimum value from each observation and then dividing by the actual range of that variable before entering them into the model. We used a 2-step conditional probit model to model the effect of the aforementioned variables on the outcomes. The parameter estimates from this model were then used to compute the predictive score for each outcome after the Norwood operation. Instead of modeling the probability of mortality and composite poor outcome separately, we approached this problem with a 2-step conditional probit model. We first used a bivariate (2-dimensional) outcome measure for each patient; we defined a bivariate random vector $Y=\left(Y_{1}, Y_{2}\right)$ such that they take the following values:

$$
y=\left\{\begin{array}{lc}
(0,0), & \text { Alive, No Morbidity } \\
(1,0), & \text { Dead, No Morbidity } \\
(0,1), & \text { Alive, Has Morbidity } \\
(1,1), & \text { Dead, Has Morbidity }
\end{array}\right.
$$

Thus, $Y_{1}$ was the indicator variable for mortality or heart transplant (yes/ no), and $Y_{2}$ was the indicator for morbidity, which was defined as the presence of at least 1 of the following 4 factors: (1) need for cardiopulmonary resuscitation after Norwood operation, (2) use of ECMO outside of the operating room, (3) prolonged mechanical ventilation, or (4) prolonged length of hospital stay. To model this bivariate outcome $Y$ for each patient based on preoperative characteristics, we used a 2-step conditional probit regression model. We reparametrized the joint distribution of $Y=\left(Y_{1}, Y_{2}\right)$ using the marginal distribution of morbidity and the conditional distribution of mortality, given the patient does or does not have morbidity. This conditional probit model can be motivated with the 2 latent variables, $Y_{1}^{*}$ and $Y_{2}^{*}$, as follows:

$$
Y_{k}^{*}= \begin{cases}>0, & y_{k}=1 \\ <0, & y_{k}=0\end{cases}
$$

for $k=1,2$. We modeled the prior probability distribution of these 2 latent variables with the patient characteristics identified previously. For each patient $i$, we set up the model such that:

$$
\begin{aligned}
Y_{2}^{*} & \sim N\left(x_{i}^{T} \beta_{S}, 1\right) \\
Y_{1 i}^{*} \mid Y_{2 i}^{*}>0 & \sim N\left(x_{i}^{T} \beta_{M \mid S}, 1\right) \\
Y_{1 i}^{*} \mid Y_{2 i}^{*}<0 & \sim N\left(x_{i}^{T} \beta_{M \mid N S}, 1\right)
\end{aligned}
$$

where each of $\beta_{S}, \beta_{M \mid S}, \beta_{M \mid N S}$ is a $p \times 1$ vector of regression coefficients (including intercept), $x_{i}$ is a vector of patient $i$ 's characteristics, and $x_{i}^{T}$ indicates the transpose of the vector $x_{i}$. The coefficient vectors $\beta_{S}, \beta_{M \mid S}$, and 
$\beta_{M \mid N S}$ pertain to the effect of covariates on the chance of the patient having morbidity, the chance of the patient dying given the patient has morbidity, and the chance of the patient dying given the patient does not have morbidity. Here, we allow that the chance of a patient dying when the patient has morbidity might be different than the chance of the patient dying when the patient does not have morbidity. With this model, the probabilities for the 4 combinations of the bivariate outcome $Y$ were computed based on the equations to follow:

$$
\begin{aligned}
& p_{00}=P\left(Y_{1}=0, Y_{2}=0\right)=\Phi\left(-x_{i}^{T} \beta_{S}\right) \Phi\left(-x_{i}^{T} \beta_{M \mid N S}\right) \\
& p_{01}=P\left(Y_{1}=0, Y_{2}=1\right)=\Phi\left(x_{i}^{T} \beta_{S}\right) \Phi\left(-x_{i}^{T} \beta_{M \mid S}\right) \\
& p_{10}=P\left(Y_{1}=1, Y_{2}=0\right)=\Phi\left(-x_{i}^{T} \beta_{S}\right) \Phi\left(x_{i}^{T} \beta_{M \mid N S}\right) \\
& p_{11}=P\left(Y_{1}=1, Y_{2}=1\right)=\Phi\left(x_{i}^{T} \beta_{S}\right) \Phi\left(x_{i}^{T} \beta_{M \mid S}\right)
\end{aligned}
$$

where $\Phi($.$) is the cumulative distribution function of the standard normal$ distribution given by

$$
\Phi(t)=\int_{-\infty}^{t} \frac{1}{\sqrt{2 \pi}} e^{-z^{2} / 2} d z
$$

Thus, for each patient, the Norwood scores for each of the outcomes were calculated using the probabilities described previously as follows:

$$
\begin{aligned}
& P(\text { Mortality })=p_{10}+p_{11}=\Phi\left(-x_{i}^{T} \beta_{S}\right) \Phi\left(x_{i}^{T} \beta_{M \mid N S}\right)+\Phi\left(x_{i}^{T} \beta_{S}\right) \Phi\left(x_{i}^{T} \beta_{M \mid S}\right) \\
& P(\text { Composite Poor Outcome })=p_{01}+p_{10}+p_{11}=1-p_{00} \\
& =1-\Phi\left(-x_{i}^{T} \beta_{S}\right) \Phi\left(-x_{i}^{T} \beta_{M \mid N S}\right)
\end{aligned}
$$

The MCMC algorithm used to estimate the parameter vectors $\beta_{S}, \beta_{M \mid S}$, and $\beta_{M \mid N S}$ and to calculate the scores is described in detail in Appendix E1. We also devised a method to calculate the risk of in-hospital mortality associated with the Norwood operation before the actual heart operation. Before the Norwood operation, a patient's demographic and preNorwood characteristics are known (and fixed), but the variables related to the heart operation are still unknown. To overcome this hurdle, we computed a weighted average of mortality scores, averaging all possible combinations of these variables coupled with a known set of preoperative characteristics. This was achieved via a kernel-density approach based on the variables related to the heart operation. ${ }^{14}$ Please refer to the Appendix E1 for more details regarding the kernel density method.

Model validation. We used an internal 10-fold cross-validation approach to validate our prediction tool. Ten subsets (approximately 1/ 10th) of the data were created at random. The first subset was held out as the test sample, and the model was fitted on the remaining 9 subsets combined (training set). The resulting score developed from this training sample was used to predict outcomes in the test set. This process was repeated 10 times overall, holding each subsequent subset as a test sample and using the rest of the data as the training sample. To assess the accuracy of the prediction model, we calculated the area under the received operating characteristic curve for each outcome on the combined validation sample from the 10 rotations. Finally, we created a Web-based tool using R Shiny to allow users to calculate risk scores for individual patients. ${ }^{15}$ In general, 2-sided $P$ values less than or equal to .05 were considered to be statistically significant.

\section{RESULTS}

In total, 549 patients qualified for inclusion. Of these, 241 patients $(43.9 \%)$ were associated with composite poor outcome. In-hospital mortality was noted among 88 patients $(16.0 \%)$, and cardiopulmonary resuscitation was required for 97 patients $(17.6 \%)$ after heart operation. ECMO was deployed for 35 patients $(6.4 \%)$ in the operating room and for 56 patients $(10.3 \%)$ outside of the operating room. The median duration of mechanical ventilation was 7 days (interquartile range 5,13 ), and the median duration of hospital stay was 24 days (interquartile range 16, 41).

The characteristics of patients with and without composite poor outcomes are shown in Table 1. The patients associated with composite poor outcomes were smaller in weight, associated with lower gestational age, more commonly associated with obstructed venous return, and were intubated more frequently before their Norwood operation. Operation characteristics such as longer cardiopulmonary bypass time, longer crossclamp time, open sternum after the heart operation, ECMO deployment at termination of heart operation in the operating room, and postoperative tricuspid valve regurgitation were associated more commonly with composite poor outcome. In addition, lower site volume and lower surgeon experience also were associated with a greater incidence of composite poor outcomes (Table 1).

We then used a Bayesian logistic lasso regression model to select variables for inclusion in the final model for creating the score. The training dataset used 520 patients to make the prediction model. The following variables were selected for inclusion by the variable selection model: birth weight, sex, gestational age, race, hypoplastic left heart syndrome, obstructed venous return, mechanical ventilation before Norwood operation, cardiopulmonary bypass time, crossclamp time, type of shunt (MBT shunt or RVPA shunt), other concomitant procedures performed at Norwood operation, ECMO deployment in the operating room, moderate/severe tricuspid valve regurgitation during Norwood operation, site volume, and surgeon experience. The following other variables were selected for inclusion in the model a priori because of their established clinical importance or on the basis of existing literature: aortic atresia, regional cerebral perfusion, and type of aortic arch reconstruction (Table 2). ${ }^{1-7,10}$ Overall, $90 \%$ of the variability among the latent variables was explained by the covariates in the final model used to create the prediction tool. An online calculator for the tool can be accessed at https://soipredictiontool.shinyapps.io/ NorwoodScoreApp/. In addition to predicting outcomes after the Norwood operation, our model also predicts mortality before the heart operation.

We then validated the model with a 10 -fold, internal cross-validation approach. Model validation was done on 520 observations. Our model predicted 92 deaths in our internal validation sample when the observed number of deaths was 88 , and it predicted 240 patients with composite poor outcome when the observed number of patients with composite poor outcomes was 238 (Table 3). Overall, the area under the received operating characteristic curve on the internal validation sample was 0.77 for mortality and 0.72 for composite poor outcome, suggesting that the model has good discriminative ability for predicting outcomes (Figure 1). 
TABLE 1. Patient and center characteristics

\begin{tabular}{|c|c|c|c|}
\hline Variable & $\mathbf{n}$ & $\begin{array}{l}\text { Composite poor } \\
\text { outcome, } \mathbf{n}(\%)\end{array}$ & $P$ \\
\hline \multicolumn{4}{|l|}{ Age at Norwood, d } \\
\hline$<5$ & 154 & $65(42.2)$ & .64 \\
\hline 5 to $<7$ & 159 & $67(42.1)$ & \\
\hline$\geq 7$ & 236 & $109(46.2)$ & \\
\hline \multicolumn{4}{|l|}{ Birth weight, $g$} \\
\hline$<2880$ & 176 & $96(54.6)$ & .002 \\
\hline 2881 to $<3334$ & 186 & $77(41.4)$ & \\
\hline$\geq 3334$ & 187 & $68(36.4)$ & \\
\hline \multicolumn{4}{|l|}{ Sex } \\
\hline Female & 209 & $90(43.1)$ & .75 \\
\hline Male & 340 & $151(44.1)$ & \\
\hline \multicolumn{4}{|l|}{ Gestational age, wk } \\
\hline$<37$ & 64 & $40(62.5)$ & .001 \\
\hline$\geq 37$ & 485 & $201(41.4)$ & \\
\hline \multicolumn{4}{|l|}{ Prenatal diagnosis } \\
\hline No & 129 & $60(46.5)$ & .49 \\
\hline Yes & 420 & $181(43.1)$ & \\
\hline \multicolumn{4}{|l|}{ Race } \\
\hline White & 436 & $190(43.6)$ & .29 \\
\hline African American & 86 & $35(40.7)$ & \\
\hline Other & 22 & $13(59.1)$ & \\
\hline Hispanic ethnicity & & & .65 \\
\hline No & 438 & $193(44.1)$ & \\
\hline Yes & 101 & 42 (41.6) & \\
\hline \multicolumn{4}{|l|}{ Anatomic diagnosis } \\
\hline \multicolumn{4}{|l|}{ HLHS } \\
\hline No & 75 & $40(53.3)$ & .08 \\
\hline Yes & 474 & $201(42.4)$ & \\
\hline \multicolumn{4}{|l|}{ Left ventricle present } \\
\hline No & 167 & 70 (41.9) & .61 \\
\hline Yes & 375 & $166(44.3)$ & \\
\hline \multicolumn{4}{|l|}{ Aortic atresia } \\
\hline No & 206 & $95(46.1)$ & .41 \\
\hline Yes & 343 & $146(42.6)$ & \\
\hline \multicolumn{4}{|c|}{ Ascending aorta diameter, $\mathrm{mm}$} \\
\hline$<2$ & 114 & $48(42.1)$ & .75 \\
\hline 2 to $<3.5$ & 231 & $101(43.7)$ & \\
\hline$\geq 3.5$ & 190 & $88(46.3)$ & \\
\hline \multicolumn{4}{|l|}{ Obstructed venous return } \\
\hline No & 530 & $226(42.6)$ & .001 \\
\hline Yes & 19 & $15(79.0)$ & \\
\hline \multicolumn{4}{|l|}{ Baseline TV regurgitation } \\
\hline No & 167 & 70 (41.9) & .66 \\
\hline Yes (moderate/severe) & 375 & $166(44.3)$ & \\
\hline \multicolumn{4}{|l|}{ Pre-Norwood interventions } \\
\hline \multicolumn{4}{|l|}{ Fetal intervention } \\
\hline No & 406 & $175(43.1)$ & .98 \\
\hline Yes & 14 & $6(42.9)$ & \\
\hline \multicolumn{4}{|l|}{ Cardiac catheterization } \\
\hline No & 521 & $224(43.0)$ & .06 \\
\hline Yes & 28 & $17(60.7)$ & \\
\hline
\end{tabular}

TABLE 1. Continued

\begin{tabular}{|c|c|c|c|}
\hline Variable & $\mathbf{n}$ & $\begin{array}{l}\text { Composite poor } \\
\text { outcome, n (\%) }\end{array}$ & $P$ \\
\hline \multicolumn{4}{|l|}{ Other surgical procedures } \\
\hline No & 541 & $234(43.3)$ & .01 \\
\hline Yes & 8 & 7 (87.5) & \\
\hline \multicolumn{4}{|l|}{ Endotracheal intubation } \\
\hline No & 284 & $108(38.0)$ & .003 \\
\hline Yes & 263 & $133(50.6 \%)$ & \\
\hline \multicolumn{4}{|l|}{ Operation characteristics } \\
\hline \multicolumn{4}{|l|}{ Total bypass time, $\min$} \\
\hline$<114$ & 180 & $65(36.1)$ & .007 \\
\hline 114 to $<159$ & 180 & $77(42.8)$ & \\
\hline$\geq 159$ & 189 & $99(52.4)$ & \\
\hline \multicolumn{4}{|l|}{ Total crossclamp time, min } \\
\hline$<43$ & 176 & $66(37.5)$ & .006 \\
\hline 43 to $<61$ & 176 & $71(40.3)$ & \\
\hline$\geq 61$ & 197 & $104(52.8)$ & \\
\hline \multicolumn{4}{|l|}{ Shunt type } \\
\hline RVPA & 281 & $118(42.0)$ & .35 \\
\hline Modified BT & 268 & $123(45.9)$ & \\
\hline \multicolumn{4}{|l|}{ Classic aortic arch repair } \\
\hline No & 66 & $28(42.4)$ & .80 \\
\hline Yes & 479 & $211(44.1)$ & \\
\hline \multicolumn{4}{|c|}{ Other concomitant cardiac procedures } \\
\hline No & 488 & $208(42.6)$ & .08 \\
\hline Yes & 61 & $33(54.1)$ & \\
\hline \multicolumn{4}{|l|}{ Use of steroids } \\
\hline No & 51 & $20(39.2)$ & .48 \\
\hline Yes & 498 & $221(44.4)$ & \\
\hline \multicolumn{4}{|l|}{ Regional cerebral perfusion } \\
\hline No & 297 & $119(40.1)$ & .04 \\
\hline Yes & 252 & $122(48.4)$ & \\
\hline \multicolumn{4}{|l|}{ Ultrafiltration } \\
\hline No & 187 & $84(44.9)$ & .72 \\
\hline Yes & 362 & $157(43.4)$ & \\
\hline \multicolumn{4}{|l|}{ Open sternum } \\
\hline No & 118 & $23(19.5)$ & $<.001$ \\
\hline Yes & 426 & $213(50.0)$ & \\
\hline \multicolumn{4}{|c|}{ Post-Norwood TV regurgitation } \\
\hline No & 364 & 115 (31.6) & .002 \\
\hline Yes (moderate/severe) & 116 & $59(50.9)$ & \\
\hline \multicolumn{4}{|c|}{ Post-Norwood neoaortic valve regurgitation } \\
\hline No & 470 & $169(36.0)$ & .51 \\
\hline Yes (moderate/severe) & 11 & $5(45.5)$ & \\
\hline \multicolumn{4}{|c|}{ ECMO use in operating room } \\
\hline No & 514 & $212(41.3)$ & $<.001$ \\
\hline Yes & 35 & $29(82.9)$ & \\
\hline
\end{tabular}

Site volume

Annual single-ventricle volume, per y

$\begin{array}{lrrr}\leq 15 & 93 & 56(60.2) & .002 \\ 16 \text { to } \leq 20 & 109 & 57(52.3) & \\ 21 \text { to } \leq 30 & 176 & 65(36.9) & \\ >30 & 171 & 63(36.8) & \\ \begin{array}{l}\text { Annual surgeon Norwood volume, per y } \\ \leq 5\end{array} & 108 & 62(57.4) & .009 \\ \leq 5 & & & \text { (Continued) }\end{array}$


TABLE 1. Continued

\begin{tabular}{lrcc}
\hline \multicolumn{1}{c}{ Variable } & n & $\begin{array}{c}\text { Composite poor } \\
\text { outcome, } \mathbf{n}(\%)\end{array}$ & $\boldsymbol{P}$ \\
\hline 6 to $\leq 10$ & 113 & $57(50.4)$ \\
11 to $\leq 15$ & 239 & $92(38.5)$ \\
$>15$ & 89 & $30(33.7)$ & \\
\hline
\end{tabular}

HLHS, Hypoplastic left heart syndrome; $T V$, tricuspid valve; $R V P A$, right ventriclepulmonary artery; $B T$, Blalock-Taussig; $E C M O$, extracorporeal membrane oxygenation.

\section{DISCUSSION}

We report a prognostic tool to predict poor outcomes both before and after a Norwood operation; the tool uses advanced modeling methods, such as Bayesian conditional probit regression models and MCMC simulations (Video 1). The newly proposed risk adjustment tool, composed of patient data, cardiac diagnosis, data related to heart operation, site volume, and surgeon experience, has a high degree of discrimination for predicting mortality and other outcomes among children undergoing a Norwood operation. This risk adjustment tool provides an efficient adjustment by condensing 10 risk factors into 1 probability before the heart operation and condensing 18 risk factors into 1 probability after the heart operation to predict poor outcomes.

To our knowledge, this is the first risk adjustment tool created to predict outcomes associated with this high-risk operation. Risk-stratifying patients before the heart operation will allow providers to deal with the technical, ethical, and financial issues inherent to this high-risk operation. This prognostic tool, which is unique to each patient, provides an opportunity for family counseling before and immediately after the heart operation. It also provides an opportunity to determine the probability of other poor outcomes related to the Norwood operation, including heart transplantation, the need for cardiopulmonary resuscitation, use of ECMO outside of the operating room, prolonged mechanical ventilation, or prolonged length of hospital stay.

Recently, Jeffries and colleagues ${ }^{16}$ developed a severityof-illness score called the Pediatric Index of Cardiac Surgical Intensive Care Mortality score for children undergoing heart operations. However, this score was designed for use with surgical operations of all complexities, and it lacks important variables related to cardiac diagnoses, heart operation, surgeon experience, and site volume. We know from existing literature that these are all important risk factors for predicting morbidity and mortality among children undergoing a Norwood operation. ${ }^{1-7,17,18}$ Hence, including these factors may better predict outcomes in a uniform patient population than previous methods.

Our study uses advanced statistical tools to discover relationships between multiple variables and outcomes. The model developed in our study is more precise, accurate, and unbiased as compared with commonly used regression models (such as logistic regression or probit regression models). Even though the logit and probit link functions are highly nonlinear, the traditional regression models use the point estimates of covariate effects. Treating the output as a point estimate of probability would result in significant approximation error. Our method does not rely on point estimates of covariate effects on outcomes. Our new model takes into account the effect of any such model uncertainty as it computes the entire probability distribution for the risk of outcome for any patient. The conditional probit approach also allows one to compute the conditional risk of any one outcome given the knowledge of other outcomes.

To determine the probability of mortality occurring before the Norwood operation is performed, we used a sophisticated model-based approach. We dealt with 2 probability distributions simultaneously: (1) the empirical distribution for the model coefficients in the probitregression as determined through MCMC and (2) the distribution for the variables related to the heart operation that cannot be learned prior to the operation. For the latter distribution, we chose a nonparametric density estimation technique based on kernels so that our method does not rely on any assumption about the pattern of those variables in the historical clinical records only. The benefit of using this probability-based approach instead of relying on point-estimates is that it takes into account many possible real-world scenarios, weighted by the likelihood of their occurrence so that the final output adequately reflects the uncertainty in inference and decision-making.

Our study has several limitations. The retrospective nature of this study renders it susceptible to study design flaws and bias. The data used to create the prediction tool were collected during a period of time when surgical and postoperative practices might have been different than the current practices. Although we attempted to adjust for important patient- and center-level factors, our dataset lacked important variables (eg, underlying comorbidities, data on postoperative variables immediately after the heart operation, use of nitric oxide, data on inotropes, and data on mechanical ventilation) that could have impacted our analysis. We also could not account for team structure or night time inhouse practitioner coverage for the intensive care unit that could have impacted the study outcomes. ${ }^{19}$

Another potential shortcoming of our study is that the dataset that was used to derive the prediction tool also was used to validate the same tool. Therefore, we recommend validating this tool in a different multi-institutional dataset from the current era. We believe there is an opportunity to improve the model discrimination with additional clinical data, and we recommend that our tool be validated over time for continued reproducibility. Like other prediction models, our tool could be subject to drift that may require frequent modifications. Because our tool was 
TABLE 2. Bayesian lasso regression variable selection model

\begin{tabular}{|c|c|c|c|c|c|}
\hline Variable & Level & Estimate & Lower 50\% & Upper $\mathbf{5 0} \%$ & Selected \\
\hline Age at Norwood, d & $1 \mathrm{~d}$ & 0.38 & -0.01 & 0.86 & No \\
\hline Birth weight, $g$ & $1 \mathrm{~g}$ & -1.57 & -2.05 & -1.09 & Yes \\
\hline Sex & Male & 0.11 & 0.01 & 0.22 & Yes \\
\hline Gestational age, wk & $1 \mathrm{wk}$ & -1.34 & -1.92 & -0.81 & Yes \\
\hline Prenatal diagnosis & Yes & 0 & -0.1 & 0.11 & No \\
\hline Race & AA & -0.11 & -0.26 & 0.01 & Yes \\
\hline Race & Other & 0.31 & 0.07 & 0.59 & Yes \\
\hline Hispanic ethnicity & Yes & 0.01 & -0.1 & 0.12 & No \\
\hline HLHS & Yes & -0.28 & -0.46 & -0.11 & Yes \\
\hline Left ventricle present & Yes & 0.05 & -0.04 & 0.16 & No \\
\hline Aortic atresia & Yes & -0.07 & -0.19 & 0.03 & No \\
\hline Exterior diameter ascending aorta & $1 \mathrm{~mm}$ & -0.1 & -0.41 & 0.18 & No \\
\hline Obstructed venous return & Yes & 0.84 & 0.43 & 1.27 & Yes \\
\hline Pre-Norwood ETT & Yes & 0.2 & 0.08 & 0.33 & Yes \\
\hline Pre-Norwood cardiac catheterization & Yes & 0.03 & -0.2 & 0.26 & No \\
\hline Baseline TV regurgitation & Moderate/severe & 0 & -0.12 & 0.14 & No \\
\hline Operation bypass time & $1 \mathrm{~min}$ & 0.6 & 0.17 & 1.12 & Yes \\
\hline Operation cross clamp time & $1 \mathrm{~min}$ & 0.79 & 0.25 & 1.37 & Yes \\
\hline Use of steroids & Yes & 0.14 & -0.01 & 0.33 & No \\
\hline Regional cerebral perfusion & Yes & -0.06 & -0.18 & 0.04 & No \\
\hline Ultrafiltration & Yes & 0.02 & -0.07 & 0.13 & No \\
\hline Shunt type & MBT shunt & 0.12 & 0.02 & 0.24 & Yes \\
\hline Type of arch reconstruction & Classic & 0.07 & -0.06 & 0.22 & No \\
\hline Other concomitant cardiac procedures & Yes & 0.22 & 0.06 & 0.41 & Yes \\
\hline ECMO use in operating room & Yes & 1.18 & 0.87 & 1.48 & Yes \\
\hline Post-Norwood TV regurgitation & Moderate/severe & 0.93 & 0.78 & 1.07 & Yes \\
\hline Post-Norwood neoaortic valve regurgitation & Moderate/severe & 0.21 & -0.08 & 0.56 & No \\
\hline Site volume & $>20$ & -0.61 & -0.78 & -0.44 & Yes \\
\hline Surgeon volume & $>10$ & -0.22 & -0.36 & -0.1 & Yes \\
\hline
\end{tabular}

AA, African-American; $H L H S$, hypoplastic left heart syndrome; $E T T$, endotracheal tube; $T V$, tricuspid valve; $M B T$, modified Blalock-Taussig; $E C M O$, extracorporeal membrane oxygenation.

derived from patients undergoing a Norwood operation, this tool should not be applied to patients undergoing other heart operations.

\section{CONCLUSIONS}

We report a novel prognostic tool to predict real-time outcomes among children undergoing a Norwood operation.

TABLE 3. Comparison of predicted with observed outcomes by quintile of predictive index for composite poor outcome

\begin{tabular}{|c|c|c|c|c|c|c|c|}
\hline \multirow[b]{2}{*}{ Quintile } & \multirow[b]{2}{*}{$\mathbf{n}$} & \multicolumn{3}{|c|}{ New tool probability } & \multicolumn{2}{|c|}{ Composite poor outcome } & \multirow{2}{*}{$\frac{\text { Incidence ratio }}{\text { O/E }(95 \% \text { CI })}$} \\
\hline & & Minimum & Maximum & Average & $\mathbf{O}$ & $\mathbf{E}$ & \\
\hline 1 & 108 & 0.0899 & 0.2308 & 0.1731 & 28 & 18.7 & $1.49(0.94-2.26)$ \\
\hline 2 & 108 & 0.2313 & 0.3393 & 0.2837 & 30 & 30.6 & $0.98(0.63-1.46)$ \\
\hline 3 & 108 & 0.3410 & 0.4501 & 0.3965 & 42 & 42.8 & $0.98(0.68-1.37)$ \\
\hline 4 & 108 & 0.4517 & 0.6376 & 0.5469 & 58 & 59.1 & $0.98(0.73-1.30)$ \\
\hline 5 & 108 & 0.6427 & 0.9999 & 0.8226 & 80 & 88.8 & $0.91(0.70-1.14)$ \\
\hline Overall & 540 & 0.0899 & 0.9999 & 0.4446 & 238 & 240.1 & $0.99(0.86-1.13)$ \\
\hline
\end{tabular}

$O$, Observed; $E$, expected; $C I$, confidence interval. 


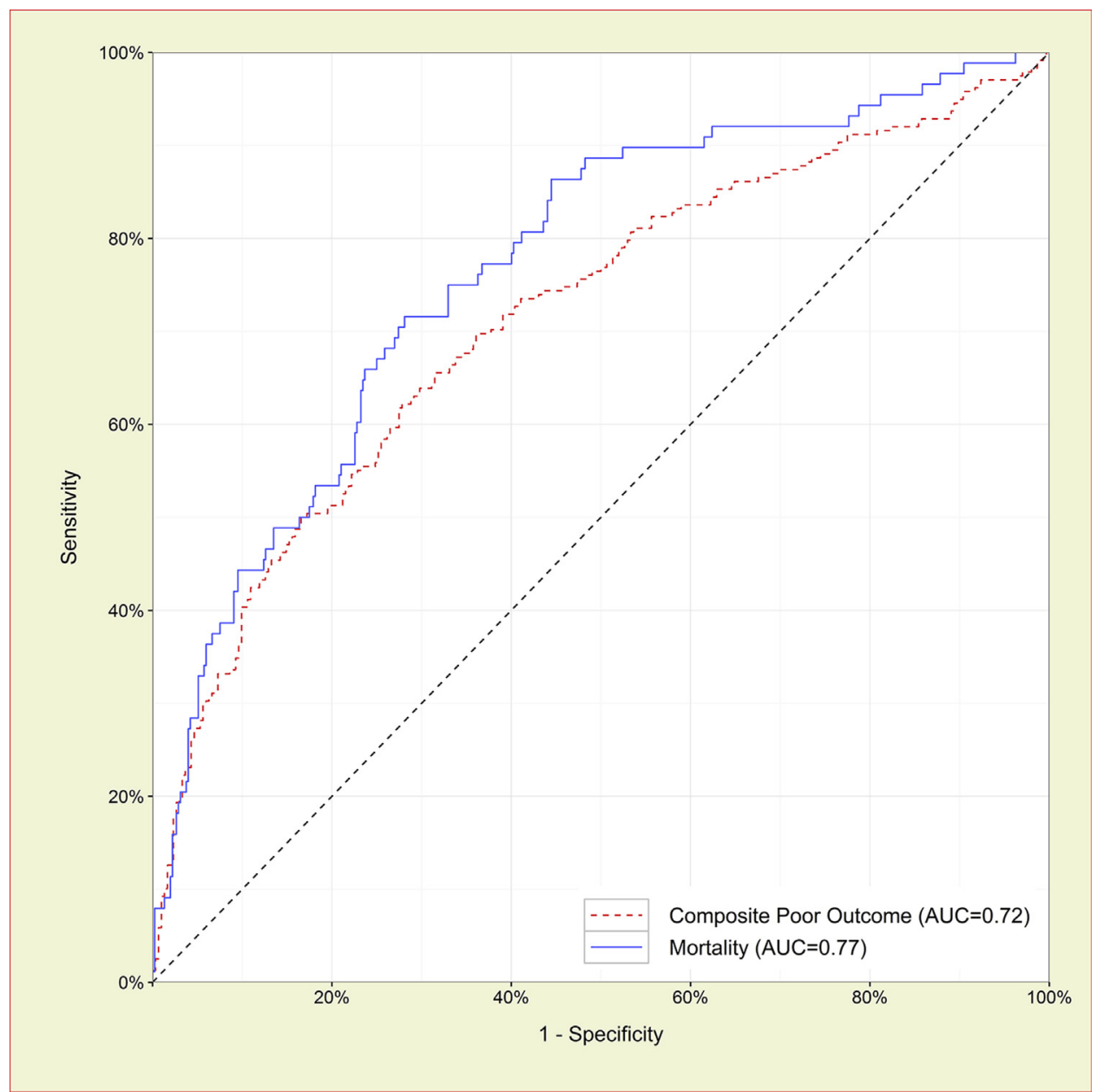

FIGURE 1. Receiver operating characteristic curves. The red line depicts the AUC for composite poor outcome, and the blue line depicts the AUC for mortality or heart transplantation. AUC, Area under the curve.

This tool is a promising first step toward creating a riskstratification for the purposes of benchmarking, family counseling, and research. In addition to providing prognostic information on mortality, this tool also provides information on other poor outcomes after the Norwood operation, such as heart transplantation, need for cardiopulmonary resuscitation, use of ECMO outside the operating

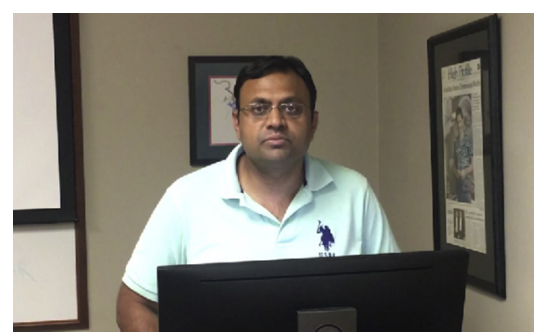

VIDEO 1. Study description by Dr Punkaj Gupta. Video available at: http://www.jtcvsonline.org/article/S0022-5223(17)31795-6/fulltext. room, prolonged mechanical ventilation, or prolonged length of hospital stay. Future studies should seek external validation and improved discrimination for this prediction tool.

\section{Conflicts of Interest Statement}

Authors have nothing to disclose with regard to commercial support.

The National Institutes of Health/National Heart, Lung, and Blood Institute Pediatric Heart Network Single-Ventricle Reconstruction Trial dataset was used to prepare this work. Data were downloaded from http://www.pediatricheartnetwork.org/For Researchers/PHNPublicUseDatasets/SingleVentricleReconstruction Trial.aspx. The authors acknowledge all of those who have contributed to the Pediatric Heart Network. The authors specifically thank Victor Zak, PhD, at New England Research Institutes for his help in working with the Pediatric Heart Network SVR Public Use Dataset. This manuscript was edited by the Science Communication Group at the University of Arkansas for Medical Sciences. 


\section{References}

1. Tabbutt S, Ghanayem N, Ravishankar C, Sleeper LA, Cooper DS, Frank DU, et al; Pediatric Heart Network Investigators. Risk factors for hospital morbidity and mortality after the Norwood procedure: a report from the Pediatric Heart Network Single Ventricle Reconstruction trial. J Thorac Cardiovasc Surg. 2012;144:882-95.

2. Tweddell JS, Hoffman GM, Mussatto KA, Fedderly RT, Berger S, Jaquiss RD, et al. Improved survival of patients undergoing palliation of hypoplastic left heart syndrome: lessons learned from 115 consecutive patients. Circulation. 2002; 106(suppl 1):I82-9.

3. Siehr SL, Maeda K, Connolly AA, Tacy TA, Reddy VM, Hanley FL, et al. Mitral stenosis and aortic atresia — a risk factor for mortality after the modified Norwood operation in hypoplastic left heart syndrome. Ann Thorac Surg. 2016; 101:162-7.

4. Kogon BE, Kanter K, Alsoufi B, Maher K, Oster ME. Outcomes and hospital costs associated with the Norwood operation: beyond morbidity and mortality. Cardiol Young. 2015;25:853-9.

5. Stasik CN, Goldberg CS, Bove EL, Devaney EJ, Ohye RG. Current outcomes and risk factors for the Norwood procedure. J Thorac Cardiovasc Surg. 2006;131:412-7.

6. Gaynor JW, Mahle WT, Cohen MI, Ittenbach RF, DeCampli WM, Steven JM, et al. Risk factors for mortality after the Norwood procedure. Eur J Cardiothorac Surg. 2002;22:82-9.

7. Sano S, Huang S, Kasahara S, Yoshizumi K, Kotani Y, Ishino K. Risk factors for mortality after the Norwood procedure using right ventricle to pulmonary artery shunt. Ann Thorac Surg. 2009;87:178-86.

8. Angus DC, Pinsky MR. Risk prediction: judging the judges. Intensive Care Med. 1997;23:363-5

9. Knaus WA, Wagner DP, Lynn J. Short-term mortality predictions for critically ill hospitalized adults: science and ethics. Science. 1991;254:389-94.

10. Ohye RG, Gaynor JW, Ghanayem NS, Goldberg CS, Laussen PC, Frommelt PC, et al; Pediatric Heart Network Investigators. Design and rationale of a randomized trial comparing the Blalock-Taussig and right ventricle-pulmonary artery shunts in the Norwood procedure. J Thorac Cardiovasc Surg. 2008;136:968-75.
11. Ohye RG, Sleeper LA, Mahony L, Newburger JW, Pearson GD, Lu M, et al; Pediatric Heart Network Investigators. Comparison of shunt types in the Norwood procedure for single-ventricle lesions. N Engl J Med. 2010;362:1980-92.

12. Wernovsky G, Wypij D, Jonas RA, Mayer JE Jr, Hanley FL, Hickey PR, et al. Postoperative course and hemodynamic profile after arterial switch operation in neonates and infants: a comparison of low-flow cardiopulmonary bypass and circulatory arrest. Circulation. 1995;92:2226-35.

13. Schmidt DF, Makalic E. bayesreg: Bayesian regression models with continuous shrinkage priors. R package version 1.0. Available at: https://CRAN.R-roject. org/package=bayesreg. Accessed August 20, 2017.

14. Scott DW. Kernel density estimation. Multivariate Density Estimation: Theory Practice, and Visualization. 2nd ed. Hoboken, NJ: Wiley; 2015:137-216.

15. Chang W, Cheng J, Allaire JJ, Xie Y, McPherson J. shiny: Web application Framework for R. R package version 0.14.1. Available at: https://CRAN.Rproject.org/package $=$ shiny. Accessed August 20, 2017.

16. Jeffries HE, Soto-Campos G, Katch A, Gall C, Rice TB, Wetzel R. Pediatric in dex of cardiac surgical intensive care mortality risk score for pediatric cardiac critical care. Pediatr Crit Care Med. 2015;16:846-52.

17. Pasquali SK, Jacobs JP, Xe H, Hornik CP, Jaquiss RD, Jacobs ML, et al. The complex relationship between center volume and outcome in patients undergoing the Norwood operation. Ann Thorac Surg. 2012;93:1556-62.

18. Nathan M, Sleeper LA, Ohye RG, Frommelt PC, Caldarone CA, Tweddell JS, et al; Pediatric Heart Network Investigators. Technical performance score is associated with outcomes after the Norwood procedure. J Thorac Cardiovasc Surg. 2014;148:2208-13.

19. Gupta P, Rettiganti M, Jeffries HE, Brundage N, Markovitz BP, Scanlon MC et al. Association of 24/7 in-house ICU attending coverage with outcomes in children undergoing heart operations. Ann Thorac Surg. 2016;102:2052-61.

Key Words: Norwood operation, prediction tool, severity of illness, risk predictors-Bayesian, Markov chain Monte Carlo 


\section{APPENDIX E1. \\ Bayesian Markov Chain Monte Carlo (MCMC) Algorithm to Estimate Parameters in the Conditional Probit Model}

We estimated the parameters $\beta_{S}, \beta_{M \mid S}$, and $\beta_{M \mid N S}$ by Gibbs sampling, a MCMC technique to simulate a sequence of observations from the posterior distributions of the parameters when direct sampling is difficult. ${ }^{\mathrm{E} 1}$ Given any patient's outcome for morbidity and mortality, $y_{1}^{*}$ and $y_{2}^{*}$ for that patient are simulated from the aforementioned normal distributions but truncated to be positive $(>0)$ or negative $(<0)$, based on whether $y_{1}$ and $y_{2}$ are 1 or 0 , respectively. Given draws of $Y_{1}^{*}$ and $Y_{2}^{*}$ for every patient, we generated MCMC samples from posterior distributions of $\beta_{S}, \beta_{M \mid S}$, $\beta_{M \mid N S}$ with the following steps:

1. Assigned multivariate normal prior distributions with a large variance to each of the parameter vectors $\beta_{S}$, $\beta_{M \mid S}$, and $\beta_{M \mid N S \text {. }}$

2. The multivariate posterior distributions of $\beta_{S}, \beta_{M \mid S}$, $\beta_{M \mid N S}$ were then derived based on the conjugate normal family. ${ }^{\mathrm{E} 2}$ For the first iteration, we started with initial parameter estimates of $\beta=\left(\beta_{S}, \beta_{M \mid S}, \beta_{M \mid N S}\right)$.

3. For the $t^{\text {th }}$ iteration, we generated $y_{1}^{*(t)} \beta^{(t)}, X, y$ and $y_{2}^{*(t)} \mid \beta^{(t)}, X, y$ based on the truncated normal densities described previously.

4. Sampled $\beta^{(t+1)} \mid y_{1}^{*(t)}, y_{2}^{*(t)}, X, y$ from the posterior multivariate normal density derived in step 2 .

5. Steps 3 to 5 were repeated 75,000 times. ${ }^{\mathrm{E} 3 \mathrm{E} 4}$ The first 25,000 were then discarded as "burn-in." The remaining 50,000 samples, taken to be approximate random samples from the posterior densities of the parameters, were thinned at every fifth draw (to further reduce any correlation among samples) to obtain the final 10,000 samples of $\beta_{S}, \beta_{M \mid S}$, and $\beta_{M \mid N S}$.

6 . For each posterior sample of the parameters thus obtained, the scores for each outcome were computed with the formulae given previously.

7. The scores were then averaged over the 10,000 samples to obtain the final Norwood scores for each of the 3 outcomes.

8. For computing the risk of mortality or heart transplantation prior to the Norwood operation, we used 5000 MCMC samples from the posterior distribution for each weighted combination of the variables related to the heart operation.

\section{Assessing Convergence of the MCMC Samples}

We assessed convergence of the MCMC chain using Geweke's convergence diagnostic with the coda package in R. ${ }^{\mathrm{E} 5, \mathrm{E} 6}$ It computes a $\mathrm{z}$ score based on the difference between the first $10 \%$ and the last $50 \%$ of the posterior samples. We chose the samples from negative 2 times the logarithm of the posterior density as a way of summarizing the convergence of all parameters involved in the model. ${ }^{\mathrm{E7}} \mathrm{We}$ obtained a $\mathrm{z}$ score of -0.021 , indicating the chain had satisfactorily converged.

\section{Kernel Density Approach for Estimating Risk of Mortality Before the Heart Operation}

Before the Norwood operation, a patient's demographic, and pre-Norwood characteristics are known (and fixed), but the variables related to the heart operation are still unknown. To overcome this hurdle, we computed a weighted average of scores of mortality, averaged over all possible combinations of these variables and coupled with a known set of preoperative characteristics. This was achieved using a kernel-density approach on the space of variables related to the heart operation. ${ }^{\mathrm{E} 8}$ For the 2 continuous variables, crossclamp time and bypass time, we fitted a bivariate distribution using a Gaussian kernel as in

$$
\widehat{f}(z \mid \Sigma)=\frac{1}{n} \sum_{i=1}^{n} \phi\left(z ; z_{i}, \Sigma\right),
$$

where $n$ is the number of observations, $z$ is the 2 dimensional vector (crossclamp time, bypass time), and $z_{i}$ is the value of $z$ for the $i^{\text {th }}$ observation in the data. The kernel is a 2-dimensional Gaussian density function with mean vector $z_{i}$ and dispersion matrix $\Sigma$ evaluated at the point $z$.

$$
\phi\left(z ; z_{i}, \Sigma\right)=\frac{1}{2 \pi}|\Sigma|^{-0.5} \exp \left\{-\frac{\left(z-z_{i}\right)^{T} \Sigma^{-1}\left(z-z_{i}\right)}{2}\right\}
$$

This measures the weight of the information $z_{i}$ in determining the density at $z$ using the proximity between $z$ and $z_{i}$. The weight depends on the parameters of the kerneldispersion $\Sigma$, usually referred to as bandwidth parameters in kernel-based techniques. Larger values along the diagonal of $\Sigma$ usually result in observations farther away from $z$ being given greater importance in determining $\widehat{f}(z \mid \Sigma)$ whereas smaller bandwidth parameters lead to more localized density estimation. We estimated the entries of $\Sigma$ using plug-in bandwidth selectors. ${ }^{\text {E9 }}$ After estimating density, we evaluated $\widehat{f}(z \mid \Sigma)$ at 13 different sample quantiles and determined the weights based on normalized values of the kernel density at those points. For the 6 binary variables related to the heart operation, we chose a 6-dimensional kernel that assigns weight to every combination of these variables based on its proportion of occurrence in the observed sample. Hence, we obtained a set of weights for several different combinations of these eight variables related to the heart operation. The kernel-based approach for calculating the weights of continuous variables has been implemented with the $k s$ package in $\mathrm{R}$ software. ${ }^{\mathrm{E} 10}$

The bandwidth parameters play a key role in deciding the smoothness of the kernel density estimate for the 
continuous operative variables. Hence, selection of bandwidths assumes importance. For this work, we used the bandwidth selection technique of Wand and Jones. ${ }^{\text {E9 }}$ Here, $\Sigma=\left[\begin{array}{ll}\sigma_{11} & \sigma_{12} \\ \sigma_{21} & \sigma_{22}\end{array}\right]$ is a $2 \times 2$ symmetric positive definite matrix $\left(\sigma_{21}=\sigma_{12}\right)$. The diagonal entries $\sigma_{11}$ and $\sigma_{22}$ control the extent of local averaging in constructing the density estimates for crossclamp time and bypass time, respectively. The off-diagonal entry $\sigma_{12}$ allows for sharing of information between the 2 variables based on the strength of their association. Using a simpler diagonal structure for $\Sigma$ would be equivalent to independently fitting 2 separate kernel densities to crossclamp time and bypass time, but given a significant value of correlation coefficient $(r=0.63)$ between these 2 variables in our sample, it would not be a reasonable assumption. The plug-in method used here minimizes the mean integrated squared error (MISE, defined as $\left.\operatorname{MISE}(\Sigma)=E \int_{z}(f(z)-\widehat{f}(z \mid \Sigma))^{2} d z\right)$ as a function of $\Sigma$, where $f$ is true bivariate joint density of cross clamp time and bypass time. This method starts with an initial bandwidth matrix (referred to as the pilot bandwidth) that is plugged-in to create a closed-form expression for asymptotic MISE, and $\Sigma$ is obtained by numerically minimizing that expression. Alternatively, bandwidth selection also can be performed with cross-validation-based techniques.

\section{Percent of Variability Explained by the Model}

The percentage of variability in the outcomes that is captured by the predictor variables is particularly intuitive for a Bayesian framework. This measure is based on the continuous latent variable representation of binary data, which is what we used in our model to create the score through $Y^{*}=\left(Y_{1}^{*}, Y_{2}^{*}\right)$. The measure is given by the ratio of variability in the covariatedependent mean to the total variability of these latent variables. We obtained an empirical distribution of this measure with MCMC simulations. The mean of this measure was 0.90 , or $90 \%$, suggesting that there is a significant amount of variability among $Y^{*}$ that is explained by the covariates.

\section{E-References}

E1. Gelfand AE, Smith AF. Sampling-based approaches to calculating marginal densities. J Am Stat Assoc. 1990;85:398-409.

E2. Holmes CC, Held L. Bayesian auxiliary variable models for binary and multinomial regression. Bayesian Anal. 2006;1:145-68.

E3. Chen MH, Shao QM, Ibrahim JG. Monte Carlo Methods in Bayesian Computation. New York: Springer Science \& Business Media; 2012:1-353.

E4. Gelfand AE. Gibbs sampling. J Am Stat Assoc. 2000;95:1300-4.

E5. Geweke J. Evaluating the accuracy of sampling-based approaches to the calculation of posterior moments. Bayesian Stat. 1992;169-93.

E6. Plummer M, Best N, Cowles K, Vines K. CODA: Convergence Diagnosis and Output Analysis for MCMC. R News. 2006;6:7-11.

E7. Cowles MK, Carlin BP. Markov chain Monte Carlo convergence diagnostics: a comparative review. J Am Stat Assoc. 1996;91:883-904.

E8. Scott DW. Kernel Density Estimation. Multivariate Density Estimation: Theory, Practice, and Visualization. 2nd ed. Hoboken, NJ: Wiley; 2015: 137-216.

E9. Wand MP, Jones MC. Multivariate plug-in bandwidth selection. Comput Stat. 1994;9:97-116.

E10. Duong T. ks: kernel density estimation and kernel discriminant analysis for multivariate data in R. J Stat Softw. 2007;21:1-16. 